\title{
Heart Failure With Preserved Ejection Fraction in Children
}

\author{
- Hormonal Imbalance Between Aldosterone \\ and Brain Natriuretic Peptide -
}

Satoshi Masutani, MD; Hirofumi Saiki, MD; Clara Kurishima, MD; Hirotaka Ishido, MD; Masanori Tamura, MD; Hideaki Senzaki, MD

\begin{abstract}
Background: There is no information on heart failure (HF) with preserved ejection fraction (HFpEF, EF >50\%) in children.

Methods and Results: Through a retrospective review of 3,907 pediatric patients with cardiovascular disease, we examined the characteristics of pediatric HFpEF over a 10-year period. We identified 18 patients with HFpEF (0.5\%). They were predominantly young children (1.1 \pm 0.9 years, no sex preponderance), who had undergone surgery for congenital heart disease. They also had concentric hypertrophy and diastolic dysfunction with elevated blood pressure. Notably, HFpEF patients had more pronounced elevation of serum aldosterone but less pronounced elevation of plasma brain natriuretic peptide (BNP) than 22 systolic HF patients (SHF, EF $\leq 50 \%$ ) (aldosterone: 1,375 $\pm 1,200$ vs. $511 \pm 563 \mathrm{pg} / \mathrm{ml}, \mathrm{P}<0.05$, and BNP: $101 \pm 141 \mathrm{vs.} 749 \pm 818 \mathrm{pg} / \mathrm{ml}, \mathrm{P}<0.005)$. Consequently, the aldosterone/BNP ratio was significantly higher in HFpEF $(38 \pm 63)$ than in SHF $(1.7 \pm 1.9, \mathrm{P}<0.05)$, and an aldosterone/BNP ratio of 10.3 or higher best predicted HFpEF (area under the curve=0.89). The HF mortality rate was significantly lower in the HFpEF than in the SHF cases, and HF symptoms showed amelioration in $61 \%$ of patients during the follow-up period of $4.2 \pm 2.6$ years.

Conclusions: HFpEF does exist in children. A common pathophysiology underlies childhood and adult HFpEF despite considerable epidemiological and etiological differences. Future controlled studies are warranted to assess the cause-effect relationship between unique hormonal profiles and HFpEF. (Circ J 2013; 77: 2375-2382)
\end{abstract}

Key Words: Aldosterone; Brain natriuretic peptide; Diastolic heart failure

$\mathbf{R}$ educed ejection fraction (EF) has traditionally been used to represent heart failure (HF) syndromes, but it is now widely acknowledged that nearly half of HF patients have preserved $\mathrm{EF}(\mathrm{HFpEF})$. The diagnosis of $\mathrm{HFpEF}$ requires the following conditions to be satisfied: (1) signs or symptoms of HF; (2) normal or mildly abnormal systolic left ventricular (LV) function; (3) evidence of diastolic LV dysfunction. ${ }^{1}$ Epidemiological studies have suggested a high prevalence of $\mathrm{HFpEF}$ in adults (population prevalence: $1.1-5.5 \%$ ). ${ }^{2}$ The incidence of HFpEF has increased over the past decades, though the mortality rate associated with this disorder has remained unchanged. ${ }^{3}$

\section{Article p 2249}

With this has come an intense interest in and the need to unravel this disease, and numerous studies have enhanced our understanding of $\mathrm{HFpEF}$, including its epidemiology, 2,4 clinical characteristics, ${ }^{5}$ pathophysiology, ${ }^{6}$ and prognosis. $., 4,7-10$ Therapeutic agents established for $\mathrm{HF}$ with reduced $\mathrm{EF}$ have proved unsuccessful in patients with HFpEF. ${ }^{11}$

However, all studies on this topic have been conducted in adults, and there is virtually no information regarding $\mathrm{HFpEF}$ in children. Whether this pathological entity even exists in children is unknown. Therefore, we performed a study to define the prevalence of $\mathrm{HFpEF}$ in children, and to determine the demographic, clinical, hemodynamic, hormonal and prognostic characteristics of HFpEF in pediatric patients managed at a single institution over a 10-year period.

Received October 10, 2012; revised manuscript received March 28, 2013; accepted April 19, 2013; released online May 31, 2013 Time for primary review: 33 days

Department of Pediatric Cardiology, Saitama Medical Center, Saitama Medical University, Kawagoe, Japan

An abstract of this manuscript was presented at the annual American Heart Association Meeting in Florida, 2009.

Mailing address: Hideaki Senzaki, Professor, MD, FAHA, Staff Office Building 101, Department of Pediatric Cardiology, Saitama Medical Center, Saitama Medical University, 1981 Kamoda, Kawagoe 350-8550, Japan. E-mail: hsenzaki@ saitama-med.ac.jp

ISSN-1346-9843 doi:10.1253/circj.CJ-12-1271

All rights are reserved to the Japanese Circulation Society. For permissions, please e-mail: cj@j-circ.or.jp 


\section{Methods}

\section{Patients}

All medical records of patients referred to the Department of Pediatric Cardiology at Saitama Medical University, between January 1, 2000 and December 31, 2009, were analyzed retrospectively to identify pediatric patients $(<18$ years old) with HFpEF. HFpEF was defined as (1) HF signs or symptoms with LVEF $>50 \%$ and (2) objective evidence of diastolic dysfunction obtained by echocardiography and catheterization study; abnormal mitral inflow pattern on echocardiography $(\mathrm{E}<\mathrm{A})$ or elevation of LV end-diastolic pressure (EDP $\geq 10 \mathrm{mmHg}$ ) or central venous pressure (CVP $\geq 8 \mathrm{mmHg}$ ). The cut-off values for EDP and CVP were determined as means +2SD of the control patients in this study.

Clinical signs and symptoms of HF were based on a modification of the previously described criteria in adults: ${ }^{1}$ history of acute pulmonary edema, or the presence of at least 2 of the following clinical features with no other identifiable cause and improvement following diuresis: dyspnea on exertion/milk feeding, bilateral edema of the lower extremities, or hepatomegaly. Lung diseases, particularly chronic forms, were carefully screened and ruled out as noncardiac causes of symptoms by both chest radiography and medical history. Patients presenting with HF signs or symptoms that were associated with increased pulmonary blood flow or atrioventricular regurgitation (>grade II by echocardiography) were excluded. Other exclusion criteria were being within 2 months of surgical correction of cardiovascular lesions, univentricular circulation, ${ }^{12}$ hypertrophic/restrictive cardiomyopathies, and chromosomal abnormalities.

To better characterize HFpEF in children, we also sought to identify $\mathrm{HF}$ patients with reduced $\mathrm{EF}(\leq 50 \%)$, categorized as systolic $\mathrm{HF}$ (SHF), and compare their data with those of $\mathrm{HFpEF}$ cases. The exclusion criteria for HFpEF were also applied when identifying SHF patients. To provide the reference values for the data from echocardiography, catheterization and HF-related hormones, 20 control patients (16 with Kawasaki disease free of coronary artery lesions, 2 normal subjects who were initially suspected to have cardiac lesions, and 2 patients with small patent ductus arteriosus with pulmonary-to-systemic flow ratio $<1.1$, within an age range relatively matched to that of the $\mathrm{HFpEF}$ patients, were also randomly selected from the database. Written informed consent for cardiac catheterization was given by the parents of all patients, and this study was approved by the institutional review board of Saitama Medical University.

\section{Data Extraction}

Data regarding patient demographics, echocardiographic examination, and laboratory measurements including HF-related hormones were extracted from the medical records 1 day prior to (demographics, echocardiograms and laboratory data except HF-related hormones) or at the time of catheterization. As is typically the case, catheterization was performed when the patients were clinically stable and on adequate diuresis. Similarly, data from patients who did not undergo catheterization were extracted after they were clinically stable and on adequate diuresis. Demographic data collected were age, sex, and body size (weight, height and surface area), as well as underlying cardiovascular disease. z-values for body weight and height were calculated using the Japanese national survey data of $2001{ }^{13}$ Therapeutic background, including medications, were also extracted. Echocardiographic data on ventricular posterior wall thickness and chamber diameters of the LV measured in the parasternal long-axis view, EF calculated by Teichholtz's method and Doppler mitral inflow pattern were also collected. The relative wall thickness and LV mass index were calculated as reported previously. ${ }^{14,15}$ Ventricular EDP, relaxation time constant $(\tau)$ and ventricular diastolic stiffness data were extracted from the cardiac catheterization database. Tau was calculated using a hybrid logistic model, ${ }^{16,17}$ as well as a monoexponential model with a non-zero asymptote. ${ }^{18,19} \mathrm{LV}$ chamber stiffness was calculated using the following equation: [LV chamber stiffness $=($ LVEDP - minimal diastolic pressure $) /$ stroke volume index]. To evaluate the hormonal status of HF, plasma levels of brain natriuretic peptide (BNP) and angiotensin II, and serum levels of aldosterone were extracted from the institutional laboratory database together with other laboratory data that might be relevant to the HF condition, including serum levels of sodium, potassium, albumin, blood urea nitrogen, and creatinine. Blood samples were drawn from the inferior vena cava in patients undergoing cardiac catheterization and from peripheral veins in other patients. Lastly, prognosis was evaluated by examining the mortality rate for all causes of death, including HF. Heart transplantation was included among the HF-related mortalities.

\section{Statistical Analysis}

Data were summarized as means $\pm S D$. Differences between groups were compared by unpaired t-test for continuous variables and by Fisher's exact test for categorical variables. Receiver-operator characteristic (ROC) curves were generated to test the sensitivity and specificity of the aldosterone/BNP ratio for characterizing HFpEF in comparison with SHF. The overall survival rate was estimated by the Kaplan-Meier method and differences in survival between groups or times were tested by the log-rank test. A P value $<0.05$ was considered statistically significant.

\section{Results}

In total, 3,907 patients were referred to our department because of cardiovascular lesions from 2000 through 2009. Among these patients, we identified 18 with $\mathrm{HFpEF}(0.5 \%)$ and 22 with $\operatorname{SHF}(0.6 \%)$ who met the inclusion/exclusion criteria. The demographic data of these patients are summarized in Table 1. There was no sex difference in the prevalence of HFpEF. The mean age of the HFpEF patients $(1.1 \pm 0.8$ years) was significantly lower than that of the SHF patients. Both groups of HF patients exhibited growth retardation as indicated by markedly negative z-values for body weight and length. Notably, the zvalues were smaller in $\mathrm{HFpEF}$ patients than in SHF patients $(\mathrm{P}<0.05)$, indicating more severe growth retardation or cachexia in HFpEF than in SHF. Restriction of water intake was required in half of both groups of HF patients. A variety of medications were prescribed for each group of patients, but no significant difference between the 2 groups was observed in the details of these medications, except for dosages of loop diuretics (Table 1).

\section{Echocardiographic and Hemodynamic Data}

Echocardiographic and catheterization data are summarized in Table 2. The mean LV end-diastolic dimension of HFpEF patients was comparable to that of the controls $(100 \pm 20 \%$ of the normal reference value), indicating normal ventricular size. This was in sharp contrast to the marked LV dilation in SHF $(146 \pm 38 \%, \mathrm{P}<0.0001$ vs. HFpEF). The LV mass index was higher in both HFpEF and SHF than in controls, but the relative wall thickness was significantly higher in HFpEF than in $\mathrm{SHF}$, indicating concentric hypertrophy in $\mathrm{HFpEF}$ patients. The 


\begin{tabular}{|c|c|c|c|}
\hline & HFpEF & SHF & Control \\
\hline No. of patients & 18 & 22 & 20 \\
\hline Male sex (\%) & $8(44)$ & $15(68)$ & $13(65)$ \\
\hline Age (years) & $1.1 \pm 0.8^{*, \dagger}$ & $8.4 \pm 6.3^{\dagger}$ & $1.9 \pm 1.0$ \\
\hline Range & $(0.17-3.1)$ & $(0.003-17.6)$ & $(0.64-3.9)$ \\
\hline Body weight $(\mathrm{kg})$ & $6.6 \pm 2.2^{*, \dagger}$ & $26.9 \pm 18.8^{\dagger}$ & $11.2 \pm 2.8$ \\
\hline Body weight (z-value) & $-2.6 \pm 1.4^{*, \dagger}$ & $-1.2 \pm 1.8^{\dagger}$ & $0.0 \pm 1.0$ \\
\hline Body length $(\mathrm{cm})$ & $67 \pm 12^{*, \dagger}$ & $118 \pm 42^{\dagger}$ & $82 \pm 11$ \\
\hline Body length (z-value) & $-2.7 \pm 2.8^{\star}, \dagger$ & $-1.2 \pm 1.9$ & $-0.3 \pm 1.3$ \\
\hline Body surface area $\left(\mathrm{m}^{2}\right)$ & $0.34 \pm 0.09^{*, \dagger}$ & $0.92 \pm 0.50^{\dagger}$ & $0.49 \pm 0.10$ \\
\hline Water restriction, n (\%) & $9(50)$ & $11(50)$ & \\
\hline \multicolumn{4}{|l|}{ Medications } \\
\hline Furosemide/torasemide, $\mathrm{n}(\%)$ & $16(89)$ & $21(73)$ & \\
\hline (mg furosemide/m#) & $45 \pm 32^{*}$ & $23 \pm 22$ & \\
\hline Thiazide, n (\%) & 7 (39) & $4(18)$ & \\
\hline$\left(\mathrm{mg} / \mathrm{m}^{2}\right)$ & $2.9 \pm 4.4$ & $1.2 \pm 3.2$ & \\
\hline Spironolactone, n (\%) & $14(78)$ & $15(68)$ & \\
\hline$\left(\mathrm{mg} / \mathrm{m}^{2}\right)$ & $51 \pm 45$ & $32 \pm 27$ & \\
\hline Digoxin, n (\%) & $8(39)$ & $8(36)$ & \\
\hline$\beta$-blocker, $\mathrm{n}(\%)$ & $8(44)$ & $7(32)$ & \\
\hline ACEI, n (\%) & 7 (39) & $10(45)$ & \\
\hline ARB, n (\%) & $1(6)$ & $1(5)$ & \\
\hline
\end{tabular}

Data are mean \pm SD. ${ }^{*} \mathrm{P}<0.05$ between HFpEF and SHF, ${ }^{\mathrm{P}} \mathrm{P}<0.05$ vs. control. ${ }^{*} 1 \mathrm{mg}$ torasemide was converted to $5 \mathrm{mg}$ furosemide. ${ }^{19} \mathrm{ACEI}$, angiotensin-converting enzyme inhibitor; ARB, angiotensin II receptor blocker; HFpEF, heart failure with preserved ejection fraction; SHF, systolic heart failure.

\begin{tabular}{|lccc|}
\hline \multicolumn{2}{|c|}{ Table 2. Echocardiographic and Catheterization Data for Pediatric Patients With HFpEF and Those With SHF } \\
Echocardiography & HFpEF & SHF & Control \\
LV diastolic dimension (mm) & & & \\
LV ejection fraction (\%) & $24.4 \pm 6.6^{*}$ & $50.6 \pm 14.8^{\dagger}$ & $29.0 \pm 4.4$ \\
LV diastolic dimension (\% of normal) & $69.1 \pm 13.9^{*}$ & $25.9 \pm 11.7^{\dagger}$ & $70.2 \pm 7.3$ \\
Relative wall thickness & $100 \pm 20^{*}$ & $146 \pm 38^{\dagger}$ & $101 \pm 13$ \\
LV mass index (g/m².7) & $0.42 \pm 0.10^{*, \dagger}$ & $0.25 \pm 0.09^{\dagger}$ & $0.33 \pm 0.07$ \\
Peak E (cm/s) & $67 \pm 37^{\dagger}$ & $98 \pm 100^{\dagger}$ & $43 \pm 9$ \\
Peak A (cm/s) & $101 \pm 26$ & $92 \pm 32$ & $101 \pm 20$ \\
Cardiac catheterization & $73 \pm 33^{*}$ & $27 \pm 20^{\dagger}$ & $54 \pm 24$ \\
LV systolic pressure (mmHg) & & & \\
LV minimum pressure (mmHg) & $97 \pm 18^{\dagger}$ & $87 \pm 17$ & $82 \pm 7.0$ \\
LV end-diastolic pressure (mmHg) & $5.7 \pm 3.9^{*, \dagger}$ & $10.4 \pm 9.9^{\dagger}$ & $1.0 \pm 1.4$ \\
Central venous pressure $(\mathrm{mmHg})$ & $13.7 \pm 3.6^{*, \dagger}$ & $19.4 \pm 8.9^{\dagger}$ & $5.9 \pm 1.7$ \\
$\tau$ (non-zero asymptote) $(\mathrm{ms})$ & $8.6 \pm 3.7^{\dagger}$ & $11.0 \pm 7.0^{\dagger}$ & $4.1 \pm 1.6$ \\
$\tau$ (hybrid logistic) (ms) & $39.6 \pm 7.8^{*, \dagger}$ & $74.2 \pm 38.6^{\dagger}$ & $35.3 \pm 3.6$ \\
LV stiffness (mmHg/ml $\left.\times m^{2}\right)$ & $18.0 \pm 1.7^{*, \dagger}$ & $27.0 \pm 7.6^{\dagger}$ & $16.4 \pm 1.6$ \\
\hline
\end{tabular}

Data are mean \pm SD. ${ }^{*} \mathrm{P}<0.05$ between $\mathrm{HFpEF}$ and $\mathrm{SHF},{ }^{\dagger} \mathrm{P}<0.05$ vs control. $\mathrm{E}$, peak atrioventricular inflow velocity during early diastole; A, peak atrioventricular inflow velocity during late diastole. Other abbreviations as in Table 1.

atrial filling velocity was significantly higher in HFpEF than in $\mathrm{SHF}$.

Catheterization data were obtained for all patients with HFpEF and 8 of the patients with SHF. Systolic pressure was significantly higher in HFpEF patients than in controls and in SHF patients with age taken into consideration (multivariate regression). The EDP of both HF groups was significantly above the normal range, and the EDP elevation was more prominent in SHF than in HFpEF. Relaxation time constants were also prolonged in both HF groups as compared with the controls, but the extent of relaxation delay was smaller in HFpEF than in SHF. In contrast, ventricular stiffness was increased to a similar extent in both groups of HF patients.

\section{Laboratory Data and Hormonal Characteristics}

Table 3 summarizes the laboratory data, including HF-related hormones. Serum albumin and potassium levels were lower in SHF than in HFpEF and controls. Serum creatinine levels were slightly higher in SHF than in HFpEF. Plasma BNP levels were elevated in both HF groups, but to a significantly smaller 


\begin{tabular}{|c|c|c|c|}
\hline & HFpEF & SHF & Control \\
\hline \multicolumn{4}{|l|}{ Serum examination } \\
\hline Blood urea nitrogen (mg/dl) & $13.6 \pm 5.1^{\dagger}$ & $14.6 \pm 6.0$ & $11.3 \pm 4.0$ \\
\hline Creatinine (mg/dl) & $0.23 \pm 0.04^{*}$ & $0.41 \pm 0.21^{\dagger}$ & $0.21 \pm 0.03$ \\
\hline Albumin (g/dl) & $4.5 \pm 0.6^{*}$ & $4.0 \pm 0.6^{\dagger}$ & $4.6 \pm 0.2$ \\
\hline Sodium (mEq/L) & $138.0 \pm 3.4$ & $136.4 \pm 6.8$ & $139.5 \pm 1.8$ \\
\hline Potassium (mEq/L) & $4.7 \pm 0.6^{*}$ & $4.2 \pm 0.4$ & $4.5 \pm 0.3$ \\
\hline \multicolumn{4}{|l|}{ Hormones } \\
\hline Angiotensin II (pg/ml) & $179 \pm 353^{\dagger}$ & $91 \pm 105^{\dagger}$ & $16 \pm 10$ \\
\hline Aldosterone (pg/ml) & $1,375 \pm 1,200^{\star}, \dagger$ & $511 \pm 563^{\dagger}$ & $194 \pm 187$ \\
\hline BNP (pg/ml) & $101 \pm 141^{*, \dagger}$ & $749 \pm 818^{+}$ & $6.8 \pm 4.4$ \\
\hline
\end{tabular}

Data are mean \pm SD. ${ }^{*} \mathrm{P}<0.05$ between $\mathrm{HFpEF}$ and $\mathrm{SHF},{ }^{\mathrm{P}} \mathrm{P}<0.05$ vs. control. BNP, brain natriuretic peptide. Other abbreviations as in Table 1.
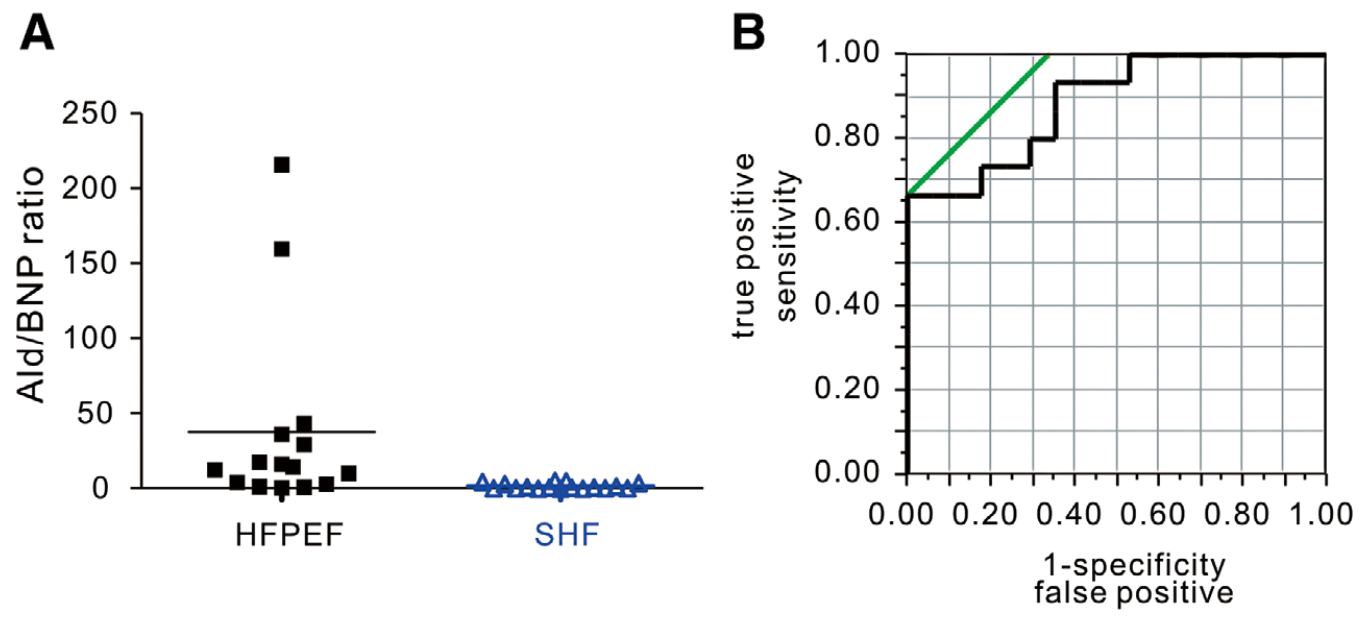

Figure 1. Hormonal status in heart failure with preserved ejection fraction (HFpEF), and systolic heart failure (SHF). (A) Ratio of serum aldosterone (ALD) to plasma brain natriuretic peptide levels (BNP) in HFpEF and SHF. ALD/BNP ratio was elevated in HFpEF, whereas it was very low in SHF. (B) Receiver-operator characteristic curve of ALD/BNP to detect HFpEF. The area under the curve was 0.89 .

degree in HFpEF than in SHF patients. The levels of both angiotensin II and aldosterone were significantly elevated in both $\mathrm{HF}$ groups as compared with controls. Angiotensin II tended to be higher in HFpEF than in SHF, but the difference did not reach statistical significance. Notably, the serum aldosterone level was significantly more elevated in HFpEF than in SHF. These data suggested an imbalance between aldosterone and $\mathrm{BNP}$ in HFpEF. Indeed, the aldosterone/BNP ratio was markedly higher in HFpEF than in $\mathrm{SHF}(38 \pm 63$ vs. $1.7 \pm 1.9, \mathrm{P}<0.05$, Figure 1A). ROC curve analysis showed that an aldosterone/ $\mathrm{BNP}$ ratio of 10.3 or higher best predicted HFpEF (area under the curve $[\mathrm{AUC}]=0.89$ ), with a sensitivity of 0.67 and specificity of 1.0 (Figure 1B).

Doses of loop diuretics were higher in HFpEF than in SHF patients (Table 1), and this may have been related to the more pronounced elevation of aldosterone in HFpEF than in SHF. Therefore, we selected $14 \mathrm{HFpEF}$ patients and $16 \mathrm{SHF}$ patients with matched diuretic doses $\left(32 \pm 18\right.$ vs. $32 \pm 20 \mathrm{mg} / \mathrm{m}^{2}$ for furosemide, $1.4 \pm 2.6 \mathrm{vs}$. $1.6 \pm 3.7 \mathrm{mg} / \mathrm{m}^{2}$ for thiazide, $42 \pm 33$ vs. $40 \pm 26 \mathrm{mg} / \mathrm{m}^{2}$ for spironolactone, all $\mathrm{P}=\mathrm{NS}$ ), and compared their aldosterone and BNP levels. Again, we observed similar hormonal characteristics: higher aldosterone $(1,467 \pm 1,252 \mathrm{vs}$. $608 \pm 577 \mathrm{pg} / \mathrm{ml}, \mathrm{P}<0.05)$, lower BNP (44 \pm 33 vs. $642 \pm 561 \mathrm{pg} / \mathrm{ml}$, $\mathrm{P}<0.05)$, and higher aldosterone/BNP ratio ( $94 \pm 158$ vs. $1.7 \pm 1.9$, $\mathrm{P}<0.05$ ) in HFpEF than in SHF. In addition, we compared serum aldosterone levels between HFpEF patients receiving higher doses of furosemide with those taking lower doses (above and below the median furosemide dose of $40.3 \mathrm{mg} / \mathrm{m}^{2}$ ). Notably, aldosterone levels were similar in the 2 groups of $\mathrm{HFpEF}$ patients $(1,420 \pm 1,431$ vs. $1,317 \pm 928 \mathrm{pg} / \mathrm{ml}$, low- vs. high-dose group, $\mathrm{P}=0.87)$. Other diuretic doses tended to be higher in the high-dose furosemide group $\left(1.2 \pm 3.6\right.$ vs. $4.5 \pm 4.8 \mathrm{mg} / \mathrm{m}^{2}$ for thiazide, $\mathrm{P}=0.11$, and $30.9 \pm 19.5$ vs. $70.3 \pm 54.8 \mathrm{mg} / \mathrm{m}^{2}$ for spironolactone, $\mathrm{P}=0.059$ ). These data support the assumption that the more pronounced activation of aldosterone in HFpEF than in SHF was independent of diuretic dose.

\section{Underlying Conditions}

As shown in Table 4, the underlying disease in SHF patients was predominantly dilated cardiomyopathy, but others devel- 
Table 4. Underlying Heart Disease in Pediatric Patients With HFpEF and Those With SHF

\section{HFpEF $(n=18)$}

(1) po TOF \& TAPVC $(n=10)$

po TOF $(n=6)$

po TAPVC $(n=4)$

(2) po CoA/IAA, either simple or complex form $(n=6)$

po $\operatorname{CoA}(n=5)$

po IAA $(n=1)$

(3) Complicated operative course $(n=2)$

Accidental injury of coronary artery (TGA) $(n=1)$

CAVB (ccTGA) $(n=1)$

\section{SHF $(\mathbf{n}=22)$}

(1) Myocardial disease $(n=19)$

Dilated cardiomyopathy $(n=17)$

LV non-compaction $(\mathrm{n}=2)$

(2) po of $\mathrm{CHD}(\mathrm{n}=3)$

po TOF $(n=1)$

po TGA $(n=1)$

po DORV $(n=1)$

$E F$, ejection fraction; po, postoperative; TOF, tetralogy of Fallot; TAPVC, total anomalous pulmonary venous connection; CoA, coarctation of aorta; IAA, interruption of aorta; VSD, ventricular septal defect; DORV, double outlet right ventricle; TGA, transposition of great arteries; ccTGA, corrected TGA; CHD, congenital heart disease; CAVB, complete atrioventricular block. Other abbreviations as in Table 1.

\begin{tabular}{|c|c|c|}
\hline & HFpEF (+) $(n=16)$ & HFpEF (-) $(n=21)$ \\
\hline Age & $1.1 \pm 0.8$ & $1.3 \pm 0.3$ \\
\hline Body weight (z-value) & $-2.5 \pm 1.4^{*}$ & $-0.6 \pm 1.1$ \\
\hline Body length (z-value) & $-2.1 \pm 2.2^{*}$ & $-0.7 \pm 0.6$ \\
\hline \multicolumn{3}{|l|}{ Cardiac catheterization } \\
\hline LV systolic pressure $(\mathrm{mmHg})$ & $99 \pm 18$ & $90 \pm 10$ \\
\hline LV minimum pressure $(\mathrm{mmHg})$ & $6.1 \pm 3.8^{\star}$ & $2.4 \pm 2.6$ \\
\hline LV end-diastolic pressure $(\mathrm{mmHg})$ & $13.3 \pm 3.8^{*}$ & $9.6 \pm 3.1$ \\
\hline Central venous pressure $(\mathrm{mmHg})$ & $8.4 \pm 3.9$ & $6.3 \pm 3.1$ \\
\hline$\tau$ (non-zero aymptote) (ms) & $40.0 \pm 8.5^{*}$ & $34.1 \pm 4.1$ \\
\hline$\tau$ (hybrid logistic) (ms) & $18.0 \pm 1.9^{*}$ & $16.3 \pm 2.1$ \\
\hline \multicolumn{3}{|l|}{ Hormones } \\
\hline Angiotensin II (pg/ml) & $197 \pm 373^{*}$ & $23 \pm 24$ \\
\hline Aldosterone (pg/ml) & $1,485 \pm 1,247^{*}$ & $360 \pm 488$ \\
\hline Brain natriuretic peptide $(\mathrm{pg} / \mathrm{ml})$ & $109 \pm 148^{*}$ & $30 \pm 43$ \\
\hline
\end{tabular}

Data are mean \pm SD. ${ }^{*} \mathrm{P}<0.05$ between $\mathrm{HFpEF}(+)$ and HFpEF (-). The present study, in fact, demonstrated the existence of the pathological condition of HFpEF in pediatric patients. They were predominantly young children who had undergone surgery for congenital heart disease, and had concentric hypertrophy and diastolic dysfunction with unique hormonal characteristics. This dataset serves as a basis for both recognizing and understanding the pathological condition of HFpEF in children. TAPVC, total anomalous pulmonary venous connection. Other abbreviations as in Tables 1,4,5.

oped SHF after surgical correction of congenital heart diseases (CHD). In contrast, HFpEF was consistently associated with $\mathrm{CHD}$ and developed predominantly during the postoperative course (Table 4). The diseases underlying HFpEF could be categorized into 3 groups: (1) CHD associated with potentially underloaded LV preoperatively, such as tetralogy of Fallot (TOF) and total anomalous pulmonary venous connection (TAPVC), (2) CHD with coarctation/interruption of the aorta (CoA/IAA), and (3) complicated operative course [accidental injury of coronary artery $(n=1)$, complete atrioventricular block $(n=1)]$. Because most patients who suffer from the same type of CHD do not generally develop HF postoperatively, we also searched for other potential causes that could lead to HFpEF by extracting pre- and peri-operative information that might be relevant to the HF etiology. Specifically, for TOF and TAPVC patients, preoperative LV end-diastolic dimension was $89 \pm 18 \%$ of the normal reference value, which is within the acceptable range for surgical correction. The cardiopulmonary bypass times $(175 \pm 37 \mathrm{~min}$ for TOF and $101 \pm 27 \mathrm{~min}$ for TAPVC) and aortic cross-clamp times $(100 \pm 32 \mathrm{~min}$ for TOF and $45 \pm 22 \mathrm{~min}$ for TAPVC) were apparently comparable with those generally achieved in surgery for the same types of CHD. ${ }^{20}$ We also compared the data from HFpEF patients with TOF, TAPVC or CoA/IAA with those of 21 age-matched non-HFpEF patients following surgery for the same diseases, focusing especially on the serum aldosterone level (Table 5). HFpEF patients had significantly higher levels of aldosterone than non-HFpEF patients. HFpEF patients also exhibited poor physical growth and diastolic dysfunction, as well as having higher levels of angiotensin II and BNP. Thus, a high serum aldosterone level appears to be associated with HFpEF status rather than specific underlying cardiac anomalies.

\section{Prognosis and Follow-up}

During the study period, 4 patients with HFpEF died, and 9 of the 22 with SHF died $(n=7)$ or underwent heart transplantation $(\mathrm{n}=2)(22 \%$ vs. $41 \%, \mathrm{P}=0.31)$. Kaplan-Meier analysis demonstrated a lower mortality tendency in HFpEF than in SHF (Figure 2, P=0.14). Interestingly, although the mortality in SHF patients was associated with worsening of $\mathrm{HF}$ in all but 


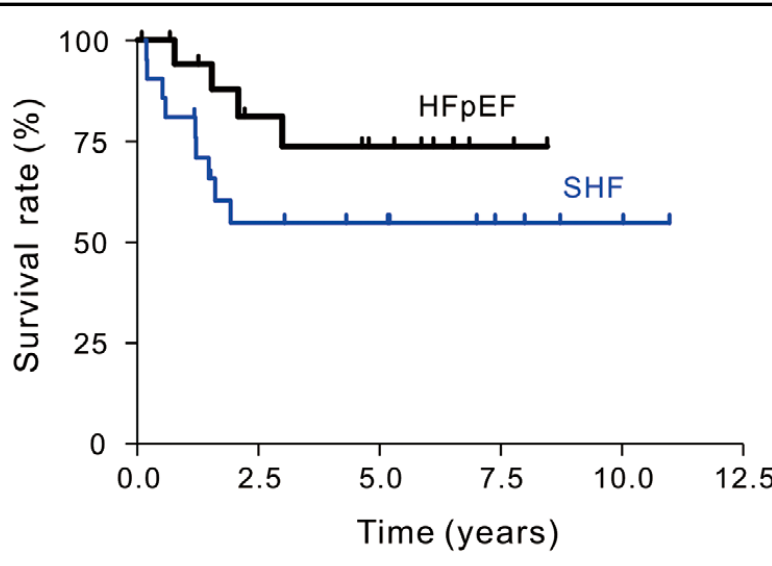

Figure 2. Kaplan-Meier curves for HFpEF and SHF. The difference in mortality between the 2 groups was borderline $(P=0.14$, by log-rank test). HFpEF, heart failure with preserved ejection fraction; SHF, systolic heart failure.

1 (sudden death) case, none of the HFpEF patients died of events directly related to HF. One patient died of pneumonia. The deaths of the remaining $3 \mathrm{HFpEF}$ patients were related to the development of new cardiac lesions (pulmonary venous obstruction in 2, aortic stenosis causing severe hemolytic anemia in 1). Thus, the HF mortality rate was significantly lower in $\mathrm{HFpEF}$ than in SHF (0\% vs. $36 \%, \mathrm{P}<0.005)$.

Of the 14 surviving patients with HFpEF, 3 remained in the state of HFpEF, and the remainder showed amelioration of $\mathrm{HF}$ symptoms during a mean follow-up period of $4.2 \pm 2.6$ years. Aldosterone antagonists were used in all 3 patients with persistent HFpEF, and in 8 of the 11 patients showing improvement.

Among the patients with $\mathrm{HFpEF}, 10$ had multiple recordings for serum aldosterone. Of these 18 patients, 3 died of newlydeveloped cardiac lesions and 1 suffered from persistent severe HF. The remaining 6 patients showed amelioration of symptoms. Serum aldosterone levels remained high in 1 patient with persistent HFpEF (4,024 to 4,326 pg/ml), but decreased in all but 1 (from $1,144 \pm 1,067$ to $394 \pm 369 \mathrm{pg} / \mathrm{ml}, \mathrm{P}=0.14$ ) of the patients showing clinical improvement.

\section{Discussion}

The present study showed a low prevalence of HFpEF in children, accounting for only $0.5 \%$ of all cohorts with possible and diagnosed cardiovascular diseases at our center over a 10-year period. These patients could be characterized as (1) being mainly young children around the age of 1 year, (2) showing severe growth retardation, (3) having a history of surgery for CHD, with some forms being relatively common, (4) ventricular size comparable with that of normal children, (5) concentric ventricular hypertrophy, (6) diastolic dysfunction, (7) elevated blood pressure, (8) distinct hormonal profile characterized by imbalance between aldosterone and BNP production, and (9) having a somewhat better prognosis with HFpEF than with SHF. These results should provide a basis for recognizing and understanding the pathological condition of HFpEF in children.

Epidemiology, Etiology and Clinical Features

The present study indicated $\mathrm{HFpEF}$ is a rare condition in chil- dren, accounting for only $0.5 \%$ of all pediatric cases with possible or diagnosed cardiovascular diseases. However, the incidence of HFpEF was comparable to that of SHF in children. Our study also indicated that HFpEF in children shows no sex preponderance, and that it occurs predominantly in infants who have undergone surgery for CHD. These patients suffered from severe growth retardation, which was especially marked in SHF patients. This is likely because of the need for water restriction in young children up to around 1 year of age when milk feeding is an important source of nutrition. Interestingly, there appeared to be a disease- and condition-preponderance for HFpEF in children: (1) CHD associated with LV underload, (2) either simple or complex forms of CoA/IAA, and (3) complicated operative events. Although these diseases and conditions have been reported to potentially have a pathophysiology that could lead to $\mathrm{HFpEF},{ }^{21-24}$ patients with similar diseases do not always develop HFpEF. Pre- and peri-operative findings of the first 2 group of patients were not particularly abnormal. In addition, HFpEF patients after surgery for TOF, TAPVC and CoA/IAA showed significantly higher levels of aldosterone, as well as more prominent clinical and hemodynamic features than non-HFpEF patients with the same diseases (Table 5). Thus, HFpEF status does not appear to be specific to the underlying cardiac anomaly.

\section{Hemodynamics and Ventricular Function}

Diastolic dysfunction is thought to be a common pathophysiological feature of HFpEF in adults, and evidence of the presence of diastolic dysfunction is included in the recently proposed diagnostic criteria for this disease. ${ }^{1}$ Our pediatric patients with HFpEF also exhibited diastolic dysfunction characterized by prolonged relaxation and increased chamber stiffness (Tables 2,5). In addition, they also had elevated systolic blood pressures as compared with SHF patients and the control subjects with age taken into consideration. Importantly, Kawaguchi et $\mathrm{al}^{25}$ demonstrated that in addition to diastolic stiffening, patients with HFpEF have systolic ventricular and arterial stiffening, which contributes not only to the hypertensive state that often accompanies HFpEF but also to exacerbation of labile blood pressure states and diastolic dysfunction. ${ }^{25}$ Interestingly, the ventricular pressure-volume coupling framework ${ }^{26}$ predicts increases in both ventricular end-systolic elastance and arterial elastance when patients have increased blood pressure under conditions of normal EF and normal ventricular size, suggesting that the children in this study with HFpEF also had systolic ventricular and arterial stiffening. Thus, the present data regarding hemodynamics and ventricular function underscore the fact that common pathophysiological features underlie HFpEF in both children and adults, despite a clear difference in etiology.

\section{Characteristics of Hormonal Activation}

Because increased ventricular wall stress and myocardial stretch are important stimuli for the production and release of natriuretic peptides, ${ }^{27}$ the difference in ventricular geometry between HFpEF and SHF (normal LV size and concentric hypertrophy vs. dilated LV and eccentric hypertrophy) would predict a smaller degree of BNP elevation in HFpEF than in SHF. The results of the present study in children were consistent with this prediction.

More importantly, we also found that in contrast to the mild elevation of BNP, serum aldosterone levels were elevated to a greater extent in HFpEF than in SHF. More pronounced increases in aldosterone levels in HFpEF appeared to be unrelated to the doses of diuretics used or specific types of under- 
lying CHD, instead being related to the pathologic condition of HFpEF per se. Although not supported by the statistical analysis, data regarding the changes in aldosterone levels with changes in clinical symptoms of HFpEF also suggested a close link between elevated aldosterone and the pathologic condition of $\mathrm{HFpEF}$. The $\mathrm{BNP}-$ aldosterone combined activation resulted in a markedly higher ratio of aldosterone/BNP in $\mathrm{HFpEF}$ than in SHF, thus differentiating HFpEF from SHF with high sensitivity and specificity. Although BNP or NT-proBNP cut-off values are used in the diagnostic algorithm for HFpEF, ${ }^{1}$ it is noteworthy that natriuretic peptide levels should be used as a supportive rather than as a definitive tool for diagnosis, mainly because of their poor specificity. The present results suggest that incorporating serum aldosterone levels may improve the diagnostic value of BNP for detecting $\mathrm{HFpEF}$, and this issue warrants further evaluation.

In addition to the diagnostic value of the serum aldosterone level and its ratio to BNP, disproportionately increased aldosterone levels relative to BNP levels may have important therapeutic implications for $\mathrm{HFpEF}$, because aldosterone promotes ventricular hypertrophy and fibrosis and exerts adverse effects on arterial structure and function by inducing inflammation and fibrosis, ${ }^{28,29}$ whereas BNP counteracts these effects of aldosterone..$^{30,31}$ Therefore, augmentation of natriuretic peptides together with aldosterone blockade may be beneficial therapeutically in this disorder. However, the present study results were at odds with this possibility, as the clinical course of HFpEF appeared to be independent of spironolactone use. Because of the retrospective nature of the present study with its small number of patients, the pathophysiological role of aldosterone and the effects of aldosterone blockade in HFpEF await clarification in future prospective controlled studies.

\section{Prognosis and Follow-up}

Our data indicated that the mortality rate in pediatric HFpEF patients was lower than that in SHF patients. In addition, death from cardiovascular causes was associated with newly-developed anatomical lesions but not with HF per se (no deaths in this series were directly associated with HF). Our data also suggested that pediatric HFpEF can be ameliorated in more than half of cases. Again, because this study was retrospective, the prognosis of patients and the effects of therapeutic agents must be clarified in future prospective studies.

\section{Study Limitations}

The present study has the limitations inherent in retrospective studies. The timing of the hormonal measurements was not strictly standardized. Duration of follow-up differed among patients and groups. Medications were administered in a noncontrolled manner. The definition of HFpEF proposed by the European study group also requires "evidence of diastolic dysfunction", particularly focusing on the utility of tissue Doppler imaging. ${ }^{1}$ We did not have tissue Doppler data, because most of our patients were referred at a time when tissue Doppler imaging was not yet available. Future studies must define the diagnostic role of tissue Doppler imaging and provide cut-off values for the diagnosis of HFpEF in children.

\section{Conclusions}

HFpEF does exist in children. Although the epidemiology, etiology and perhaps prognosis of HFpEF in children appear to be quite different from those in adults, the underlying pathophysiology represented by concentric hypertrophy, diastolic dys- function and possibly ventricular systolic and arterial stiffening appears to be the same. The novel observation of a hormonal imbalance between aldosterone and BNP in children may provide a clue to further elucidating the pathophysiology of and appropriate therapy for HFpEF. We believe that the present study provides the basis for future studies on this disease.

\section{Acknowledgments}

This study was supported by a national grant from the Japan Society for the Promotion of Science and Medical Research, Saitama Medical University Internal Grant, Grants from Nipro Corporation, Kawano Memorial Foundation, and Tenshindo Medical Institution.

We thank colleagues at our university who performed the catheterization studies.

\section{Disclosures}

None. Grant Support: A national grant from the Japan Society for the Promotion of Science and Medical Research, Saitama Medical University Internal Grant, Grants from Nipro Corporation, Kawano Memorial Foundation, and Tenshindo Medical Institution.

\section{References}

1. Paulus WJ, Tschope C, Sanderson JE, Rusconi C, Flachskampf FA, Rademakers FE, et al. How to diagnose diastolic heart failure: A consensus statement on the diagnosis of heart failure with normal left ventricular ejection fraction by the Heart Failure and Echocardiography Associations of the European Society of Cardiology. Eur Heart J 2007; 28: 2539-2550.

2. Owan TE, Redfield MM. Epidemiology of diastolic heart failure. Prog Cardiovasc Dis 2005; 47: 320-332.

3. Owan TE, Hodge DO, Herges RM, Jacobsen SJ, Roger VL, Redfield MM. Trends in prevalence and outcome of heart failure with preserved ejection fraction. N Engl J Med 2006; 355: 251-259.

4. Kitzman DW, Little WC, Brubaker PH, Anderson RT, Hundley WG, Marburger CT, et al. Pathophysiological characterization of isolated diastolic heart failure in comparison to systolic heart failure. JAMA 2002; 288: 2144-2150.

5. Zile MR, Baicu CF, Gaasch WH. Diastolic heart failure: Abnormalities in active relaxation and passive stiffness of the left ventricle. N Engl J Med 2004; 350: 1953 - 1959.

6. Kass DA, Bronzwaer JG, Paulus WJ. What mechanisms underlie diastolic dysfunction in heart failure? Circ Res 2004; 94: 1533-1542.

7. Lam CS, Donal E, Kraigher-Krainer E, Vasan RS. Epidemiology and clinical course of heart failure with preserved ejection fraction. Eur J Heart Fail 2010; 13: 18-28.

8. Little WC. Heart failure with a normal left ventricular ejection fraction: Diastolic heart failure. Trans Am Clin Climatol Assoc 2008; 119: 93-99; discussion 99-102.

9. Maurer MS, Burkhoff D, Fried LP, Gottdiener J, King DL, Kitzman DW. Ventricular structure and function in hypertensive participants with heart failure and a normal ejection fraction: The Cardiovascular Health Study. J Am Coll Cardiol 2007; 49: 972-981.

10. Zile MR, Gaasch WH, Anand IS, Haass M, Little WC, Miller AB, et al. Mode of death in patients with heart failure and a preserved ejection fraction: Results from the Irbesartan in Heart Failure With Preserved Ejection Fraction Study (I-Preserve) trial. Circulation 2010; 121: $1393-1405$.

11. Liu Y, Haddad T, Dwivedi G. Heart failure with preserved ejection fraction: Current understanding and emerging concepts. Curr Opin Cardiol 2013; 28: 187-196.

12. Ishibashi N, Park IS, Waragai T, Yoshikawa T, Murakami Y, Mori $\mathrm{K}$, et al. Effect of carvedilol on heart failure in patients with a functionally univentricular heart. Circ J 2011; 75: 1394-1399.

13. Inokuchi M, Hasegawa T, Sasaki R, Hori N, Kosaki K, Anzo M, et al. The cross-sectional BMI growth curves for Japanese from birth to 17 years of age: The 2000-2001 National Survey data: Comparison with the 1990-1994 data and the 1978-1981 data. Clin Pediatr Endocrinol 2003; 12: 122

14. Lang RM, Bierig M, Devereux RB, Flachskampf FA, Foster E, Pellikka $\mathrm{PA}$, et al. Recommendations for chamber quantification: A report from the American Society of Echocardiography's Guidelines and Standards Committee and the Chamber Quantification Writing Group, developed in conjunction with the European Association of Echocardiography, a branch of the European Society of Cardiology. J Am Soc Echocardiogr 2005; 18: 1440-1463.

15. Daniels SR, Meyer RA, Liang YC, Bove KE. Echocardiographi- 
cally determined left ventricular mass index in normal children, adolescents and young adults. J Am Coll Cardiol 1988; 12: 703-708.

16. Matsubara H, Takaki M, Yasuhara S, Araki J, Suga H. Logistic time constant of isovolumic relaxation pressure-time curve in the canine left ventricle: Better alternative to exponential time constant. Circulation 1995; 92: 2318-2326.

17. Senzaki H, Chen CH, Masutani S, Taketazu M, Kobayashi J, Kobayashi $\mathrm{T}$, et al. Assessment of cardiovascular dynamics by pressure-area relations in pediatric patients with congenital heart disease. $J$ Thorac Cardiovasc Surg 2001; 122: 535-547.

18. Raff GL, Glantz SA. Volume loading slows left ventricular isovolumic relaxation rate: Evidence of load-dependent relaxation in the intact dog heart. Circ Res 1981; 48: 813-824.

19. Senzaki H, Kamiyama MP, Masutani S, Ishido H, Taketazu M, Kobayashi T, et al. Efficacy and safety of torasemide in children with heart failure. Arch Dis Child 2008; 93: 768-771.

20. He GW. Current strategy of repair of tetralogy of Fallot in children and adults: Emphasis on a new technique to create a monocusp-patch for reconstruction of the right ventricular outflow tract. J Card Surg 2008; 23: 592-599.

21. Cheung YF, Wong SJ, Liang XC, Cheung EW. Torsional mechanics of the left ventricle in patients after surgical repair of tetralogy of Fallot. Circ J 2011; 75: 1735-1741.

22. Krymsky LD. Pathologic anatomy of congenital heart disease. Circulation 1965; 32: 814-827.

23. Iwamoto Y, Tamai A, Kohno K, Masutani S, Okada N, Senzaki H. Usefulness of respiratory variation of inferior vena cava diameter for estimation of elevated central venous pressure in children with cardiovascular disease. Circ J 2011; 75: 1209-1214.

24. Murakami T, Takeda A. Enhanced aortic pressure wave reflection in patients after repair of aortic coarctation. Ann Thorac Surg 2005; 80: 995-999.

25. Kawaguchi M, Hay I, Fetics B, Kass DA. Combined ventricular systolic and arterial stiffening in patients with heart failure and preserved ejection fraction: Implications for systolic and diastolic reserve limitations. Circulation 2003; 107: 714-720.

26. Sunagawa K, Maughan WL, Burkhoff D, Sagawa K. Left ventricular interaction with arterial load studied in isolated canine ventricle. Am J Physiol 1983; 245: H773-H780.

27. Iwanaga Y, Nishi I, Furuichi S, Noguchi T, Sase K, Kihara Y, et al. B-type natriuretic peptide strongly reflects diastolic wall stress in patients with chronic heart failure: Comparison between systolic and diastolic heart failure. J Am Coll Cardiol 2006; 47: 742-748.

28. Zannad F, Dousset B, Alla F. Treatment of congestive heart failure: Interfering the aldosterone-cardiac extracellular matrix relationship. Hypertension 2001; 38: 1227-1232.

29. Weber KT. Aldosterone and spironolactone in heart failure. $N$ Engl J Med 1999; 341: 753-755.

30. Nishikimi T, Maeda N, Matsuoka H. The role of natriuretic peptides in cardioprotection. Cardiovasc Res 2006; 69: 318-328.

31. Ito T, Yoshimura M, Nakamura S, Nakayama M, Shimasaki Y, Harada E, et al. Inhibitory effect of natriuretic peptides on aldosterone synthase gene expression in cultured neonatal rat cardiocytes. Circulation 2003; 107: 807-810. 